\title{
Escuelas de Educación Normal, Instituciones Vivenciales y Promotoras de los ODS en México
}

\section{César Antonio Acosta Fernández}

Catedrático de la Benemérita Escuela Normal Veracruzana "Enrique C. Rébsamen" de México, egresado de la Licenciatura en Educación Física en 2006 en la misma institución recibiendo la Condecoración al Mérito Estudiantil "Prof. Rafael Ramírez". Actualmente laborando con base en esta institución y desarrollándome como miembro de la subdirección administrativa, teniendo a cargo proyectos especiales para mejora y proyección de la institución para gestión e implementación. Correo electrónico cesaracosta 84@hotmail.com, celular 5212288261817.

\begin{abstract}
The 2030 agend and the SDGs are a common international goal, it is good that nations abide by them and strive to give them full compliance. Mexico signed them but apparently there is no multisectoral work, deep and organized for this purpose. Education is one of the SDGs but it is also an inert network so far, which can promote and activate any function by the SDGs. Higher education rests on the professional training of society, hence the future of a country is formed, but interestingly, it is not involved in the struggle for a worthy future. Normal education is responsible for higher education to build a the teachers and guides of the knowledge of children and for them of the hope of all people; normal education is currently reserved, when it can be the key to access a network for dissemination and implementation of small activities by the SDGs, which would be a comprehensive fulfillment of goals, adding to society and government. Sometimes it only takes a slight stimulus to drive small engines that generate great changes, I hope the normal education is this engine.
\end{abstract}

Keywords:

Education, normal education

, society, development, proposal, promotion, fulfillment.

\section{Resumen}

La agenda 2030 y los ODS son una meta internacional común, es bueno que las naciones las acaten y se esmeren por darles cabal cumplimiento. México 
las firmó pero aparentemente no hay trabajo multisectorial, profundo y organizado para este fin. La educación es uno de los ODS pero también es una red inerte hasta el momento, que puede promover y activar cualquier función por los ODS. En la educación superior se descansa la formación profesional de la sociedad, por ende ahi se forma el futuro de un país, pero curiosamente no se le involucra en la lucha por un porvenir digno. La educación normal es la responsable de la formación superior de construir a los maestros y guías del conocimiento de niños y por ellos de la esperanza de todo pueblo; al normalismo actualmente se le tiene reservado, cuando puede ser la llave para acceder a una red de difusión e implementación de pequeñas actividades por los ODS, lo que sería un cumplimiento de metas de manera integral, sumando a sociedad y gobierno. En ocasiones solo hace falta un ligero estimulo para accionar pequeños motores que generaran grandes cambios, espero la educación normal sea este motor.

\section{Palabras clave:}

Educación, normalismo, sociedad, desarrollo, propuesta, promoción, cumplimiento.

\section{Introducción}

Nuestro mundo y humanidad enfrentan grandes retos en la actualidad para lograr ser un hogar y una hermandad justa, incluyente, equitativa, segura, prospera y sostenible para todos y para cada uno de los espacios existentes. En miras de ello se han realizado diferentes esfuerzos, en distintos niveles, con tal de llegar a esa meta, todos y cada uno de 
ellos loables. El trabajo organizado, colaborativo y multisectorial es una de las claves para el logro de estos ideales, e inspirado en ello es que los líderes del mundo, en la Cumbre de las Naciones en Nueva York, en septiembre de 2015, firmaron y adoptaron el documento "Transformando nuestro mundo: la Agenda 2030 para el desarrollo sostenible", donde se incluyen los Objetivos para el Desarrollo Sostenible (ODS), debiéndose convertir para los países firmantes en la prioridad a alcanzar por sus gobiernos y políticas.

México es un país con avances significativos en el desarrollo social en el último siglo, pero con grandes pendientes referentes a los ODS. Es una nación con un valor en su gente excepcional, pero con rezagos y brechas que generan desigualdades entre los individuos en demasía marcadas, acarreando con ello problemáticas que suman y degradan cada día más a nuestro pueblo en general. Es por esto que medidas como la agenda 2030 y los ODS son planteamientos adecuados para encarrilar los esfuerzos gubernamentales, políticas públicas y ánimos de todos niveles hacia un desarrollo integral y armónico de los mexicanos.

En mi perfil como educador el ODS 4 es el que resultaría más interesante o en el que volcaría toda mi atención, pero la realidad de la educación es otra, la educación es la base y el inicio de todo, por lo que todo debe ver y partir de la educación. El objetivo cuarto de la agenda es la "Educación de calidad" y plantea metas generales de condiciones idóneas al que el servicio educativo debe llegar. Pero la educación y su sector da para mucho más, puede y debe ser promotora de todos los ODS, a partir de ella se puede alcanzar y penetrar a mas población, generar un cambio de conducta con miras a un comportamiento más responsable adoptando los principios necesarios para el cumplimiento de la agenda 2030, transformando una responsabilidad de los gobiernos a una también de todo habitante.

La división de la educación por niveles posibilita diferentes marcos de acciones, pero también genera responsabilidades sociales, siendo la Educación Superior la que centra la más alta cuantía a la agenda de mejora social. Con esto toda escuela de formación superior está moralmente obligada a crear un plan de acción para los ODS y la agenda 2030.

En México un sector muy importante, trascendente y de respeto e influencia social es el Normalismo. Es por esto que la Educación Normal debe integrarse a las acciones globales por la agenda 2030, ha de adecuar los procesos posibles para que en sus planteles los esfuerzos por los ODS sean una práctica y forma de vida común, y puede realizar proyectos específicos para la correcta y más amplia difusión de los ODS y ser promotoras de actividades que busquen su cumplimiento.

Este documento surge del trabajo que actualmente desarrollo para mi centro laboral. Se trata de un proyecto aplicado de forma piloto a partir del siguiente semestre (Sem. A 20192020), en el que se aplicará, en asignaturas y áreas administrativas, una actividad por cada ODS, buscando dar a conocer la temática, generar conciencia sobre su aplicación en la vida cotidiana y lograr prácticas que den un desarrollo sostenible a nuestro plantel. Dicha actividad no se detiene ahí, se busca también promover este proyecto y acciones ante las autoridades educativas estatales y nacionales para que su contenido sea analizado y, de ser aprobado, incluido en las reformas estructurales que se están llevando a cabo en nuestro sector, que contemplan las leyes secundarias u operativas. Con esto se quiere realizar un 
modelo de inserción y práctica cotidiana nacional de los ODS, buscando un mayor margen de penetración social mediante la educación, además de una verdadera acción para el cumplimiento del compromiso sustraído por nuestro país con la agenda 2030.

Este trabajo, en su práctica y aplicación, partirá desde la sencillez, de acciones de la vida diaria, para lograr para lograr cambios individuales y en comunidad, pretendiendo tener cabida en diferentes reglamentaciones y modelos educativos, con ello alcanzar cambios macros, ya que el actuar de cada uno es el cambio de todos.

Estoy seguro de que las escuelas son la clave para la correcta difusión y aplicación de políticas o programa públicos, en ello los maestros son los aliados natos con liderazgo natural para estas misiones. Incluir a toda escuela y docente es un paso decisivo al éxito.

\section{Justificación}

Todo esfuerzo por una noble labor es bueno y de gran importancia, no es posible ni concebible menospreciar alguno, entonces por qué no incluir verdaderamente todos los sectores de un país a una agenda de cambio por una mejor vida para la población, hacer de los ODS metas sociales y no solo gubernamentales y, sobre todo, de una acción real, tangible [ONU MUJERES (2015). La Agenda 2030 para el Desarrollo Sostenible. http://www.unwomen.org/es/what-we-do/post-2015], y no simulaciones de eventos y escritos con el fin de cumplir acuerdos y tratados de país en organismos internacionales.

Como mencioné en la introducción, estoy claro, con la apuesta máxima, de que las escuelas deberían ser la base y motor para la correcta difusión y aplicación de las políticas y programas públicos de nuestro país. Máxime el normalismo, que es de donde parten los profesionales que harán frente al reto educativo, con conocimientos y habilidades que serán los medios para incidir en cambios sociales que requiera nuestra nación [VERONICA MEDRANO CAMACHO, EDUARDO ÁNGELES MENDEZ, MIGUEL ÁNGEL MORALES HERNANDEZ. (2017). LA EDUCACIÓN NORMAL EN MÉXICO, ELEMENTOS PARA SU ANÁLISIS. México. http://publicaciones.inee.edu.mx/buscadorPub/P3/B/108/P3B108.pdf]. Por ello es que debe plantearse una iniciativa o programa específico para atender en la Educación Normal del país y desde ahí hacer una acción frontal y decidida para lograr el cumplimento de los ODS en México tomando como base su amplia y profunda estructura educativa.

Además, el principal punto de justificación es la naturaleza de la educación, el enseñar y dotar de experiencias a los educandos para lograr su desarrollo personal y profesional, pero también para su impacto e influencia en su entorno y comunidad.

Muchas personas, escépticas del tema, la han considerado como una utopía, algo que suena bien pero sin interés para su realización. Pero ese es exactamente el punto que le da entrada a los maestro para ser motores del esfuerzo, la labor del docente casi siempre es utópica si se analiza, pero es real; el maestro logra cambios y mejoras en sus educandos sin importar adversidades, recursos, lo que sea, el docente plantea sus metas y va tras ellas, con vista única en sus alumnos, con ello logrando transformaciones en sus comunidades, todo con solo su gran esfuerzo, dedicación, creatividad, ánimo, constancia y tenacidad. 


\subsection{Los números generales de la educación normal en México}

Mi país es uno de los pocos ya que conservan el sistema normalista para la enseñanza y formación de sus maestros, esto no es por un rezago ni nada por el estilo, a mi gusto es por el carácter social de la educación y la formación específica que requieren los docentes, una instrucción con una clara perspectiva teórica y metodológica simultánea. Es por esto que las escuelas normales mexicanas mantienen las siguientes características cuantitativas.

Tabla 1. Cantidad y tipo de escuelas de educación Normal en México

Escuelas normales según su tipología por sostenimiento (2015-2016)

\begin{tabular}{|c|c|c|c|}
\hline \multirow{2}{*}{ Tipología } & \multirow{2}{*}{ Total } & \multicolumn{2}{|c|}{ Sostenimiento } \\
\hline & & Público & Privado \\
\hline Escuelas Normales & 227 & 137 & 90 \\
\hline Normales Superiores & 56 & 36 & 20 \\
\hline Normales Experimentales & 23 & 23 & 0 \\
\hline Centros Regionales de Educación Normal & 16 & 16 & 0 \\
\hline Normales Rurales & 16 & 16 & 0 \\
\hline Normales Urbanas & 12 & 12 & 0 \\
\hline Centros de Actualización del Magisterio & 12 & 12 & 0 \\
\hline Escuelas Nacionales de Maestros & 2 & 2 & 0 \\
\hline Institutos & 30 & 4 & 26 \\
\hline Escuelas de Educación Física & 6 & 4 & 2 \\
\hline Escuelas de Artísticas & 1 & 0 & 1 \\
\hline Centros Escolares & 17 & 3 & 14 \\
\hline Otras (colegios, escuelas, universidades) & 42 & 1 & 41 \\
\hline Total & 460 & 266 & 194 \\
\hline
\end{tabular}

Fuente: INEE, La Educación Normal en México, Captura 2017.

Tabla 2. Alumnado por entidad federativa en el país.

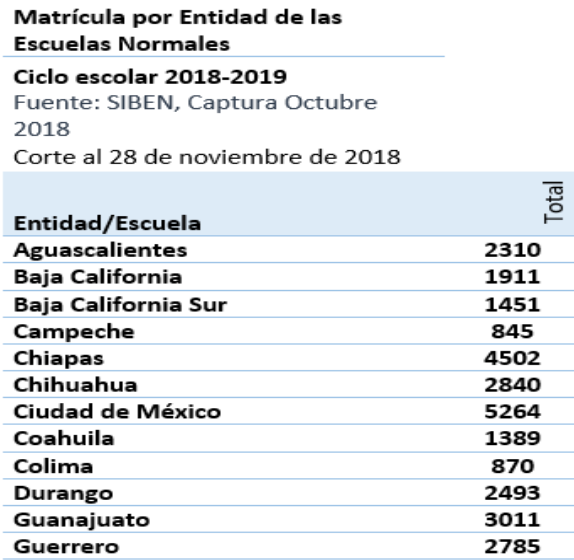

\begin{tabular}{|cc|} 
Hidalgo & 2895 \\
\hline Jalisco & 4253 \\
\hline México & 8884 \\
\hline Michoacán & 5320 \\
\hline Morelos & 1307 \\
\hline Nayarit & 1308 \\
\hline Nuevo León & 4796 \\
\hline Oaxaca & 1730 \\
\hline Puebla & 6605 \\
\hline Querétaro & 1657 \\
\hline Quintana Roo & 850 \\
\hline San Luis Potosí & 4817 \\
\hline Sinaloa & 1765 \\
\hline Sonora & 2203 \\
\hline Tabasco & 1114 \\
\hline Tamaulipas & 2157 \\
\hline Tlaxcala & 788 \\
\hline Veracruz & 3097 \\
\hline Yucatán & 3382 \\
\hline Zacatecas & 1734 \\
\hline Total general & 90333 \\
\hline
\end{tabular}

Fuente: SIBEN, Captura Octubre 2018.

\subsection{Números generales de las escuelas en México, una gran estructura si se quiere.}

Aunque en México aún faltan planteles escolares de diversos niveles y docentes que atiendan a los educandos en edad de asistir a recibir el servicio, se cuenta con un alcance interesante en todo el país, penetrando, casi mejor que cualquier otro servicio del gobierno, 
a todo el territorio nacional. El sistema educativo nacional alberga a más de la mitad de la población [SECRETARÍA DE EDUCACIÓN PÚBLICA (SEP). Sistema Interactivo de Consulta Estadistica Educativa. https://www.planeacion.sep.gob.mx/principalescifras/]

Tabla 3. Matrícula del Sistema Educativo Nacional en México.

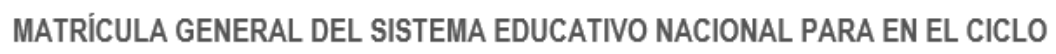

\begin{tabular}{|c|c|c|c|c|c|}
\hline Entidad & Escuelas & Alumnos & $\begin{array}{l}\text { Alumnos } \\
\text { Hombres }\end{array}$ & Alumnos Mujeres & $\begin{array}{c}\text { Docentes } \\
1\end{array}$ \\
\hline AGUASCALIENTES & 2,157 & 421,858 & 209,948 & 211,910 & 24,961 \\
\hline BAJA CALIFORNIA & 4,495 & 996,833 & 502,038 & 494,795 & 61,056 \\
\hline BAJA CALIFORNIA SUR & 1,267 & 228,556 & 111,275 & 117,281 & 13,211 \\
\hline CAMPECHE & 2,210 & 287,724 & 134,761 & 152,963 & 15,982 \\
\hline CHIAPAS & 19,644 & $1,775,668$ & 900,810 & 874,858 & 88,427 \\
\hline CHIHUAHUA & 6,736 & $1,073,899$ & 540,828 & 533,071 & 59,950 \\
\hline CIUDAD DE MÉXICO & 9,549 & $2,746,818$ & $1,386,012$ & $1,360,806$ & 191,102 \\
\hline COAHUILA DE ZARAGOZA & 5,202 & 898,883 & 450,004 & 448,879 & 51,827 \\
\hline COLIMA & 1,457 & 196,904 & 97,939 & 98,965 & 14,107 \\
\hline DURANGO & 5,616 & 535,951 & 268,533 & 267,418 & 34,761 \\
\hline GUANAJUATO & 12,220 & $1,826,427$ & 915,540 & 910,887 & 97,055 \\
\hline GUERRERO & 11,476 & $1,112,904$ & 553,125 & 559,779 & 60,480 \\
\hline HIDALGO & 8,785 & 972,082 & 478,147 & 493,935 & 57,462 \\
\hline JALISCO & 15,118 & $2,340,190$ & $1,165,424$ & $1,174,766$ & 135,669 \\
\hline MÉXICO & 22,682 & $4,643,831$ & $2,314,123$ & $2,329,708$ & 246,408 \\
\hline MICHOACÁN DE OCAMPO & 12,558 & $1,362,178$ & 670,682 & 691,496 & 82,173 \\
\hline MORELOS & 3,584 & 568,758 & 273,415 & 295,343 & 35,851 \\
\hline NAYARIT & 3,353 & 344,367 & 173,185 & 171,182 & 22,594 \\
\hline NUEVO LEÓN & 7,734 & $1,562,446$ & 825,222 & 737,224 & 83,748 \\
\hline OAXACA & 13,907 & $1,208,562$ & 592,463 & 616,099 & 71,936 \\
\hline PUEBLA & 14,906 & $2,056,490$ & $1,022,566$ & $1,033,924$ & 104,388 \\
\hline QUERÉTARO & 4,103 & 673,221 & 336,703 & 336,518 & 35,437 \\
\hline QUINTANA ROO & 2,514 & 467,177 & 226,660 & 240,517 & 25,423 \\
\hline SAN LUIS POTOSÍ & 8,576 & 880,645 & 420,141 & 460,504 & 49,769 \\
\hline SINALOA & 6,746 & $1,007,000$ & 492,048 & 514,952 & 53,549 \\
\hline SONORA & 4,769 & 851,388 & 424,770 & 426,618 & 48,345 \\
\hline TABASCO & 5,568 & 760,818 & 385,783 & 375,035 & 39,036 \\
\hline TAMAULIPAS & 6,216 & 975,461 & 491,143 & 484,318 & 58,169 \\
\hline TLAXCALA & 2,317 & 392,222 & 193,729 & 198,493 & 22,555 \\
\hline VERACRUZ DE I. DE LA LLAVE & 23,375 & $2,177,118$ & $1,096,695$ & $1,080,423$ & 134,237 \\
\hline YUCATÁN & 4,002 & 609,532 & 306,425 & 303,107 & 37,879 \\
\hline ZACATECAS & 5,152 & 494,539 & 244,140 & 250,399 & 27,543 \\
\hline TOTAL & 257,994 & $36,450,450$ & $18,204,277$ & $18,246,173$ & $2,085,090$ \\
\hline
\end{tabular}

Fuente: SEP, Sistema Interactivo de Consulta Estadística Educativa, Captura 2019.

\subsection{Líderes sociales en México, los maestros de los más importantes.}

Históricamente en México existen, por profesión, tres liderazgos que en comunidades son respetados y seguidos, con la capacidad de incidir en el pensamiento de la población y de 
motivar cambios sociales trascendentales, estos son los médicos, los representantes o líderes de cultos religiosos y los maestros. Es innegable que el valor y respeto que en México se tiene por sus docentes es alto, a lo mejor en una actual decadencia por una posible desvalorización de comunidades y familias, además de ataques pasados del gobierno, con todo el peso de los poderes de la nación, con tal de mermar su imagen ante el pueblo y así lograr imponer una mal llamada reforma educativa que solo buscaba un cambio en el manejo administrativo y financiero del sector. Los maestros son los todólogos del segundo hogar del pueblo, una imagen y labor casi paternal que normalmente los convierte en impulsores de mejoras a comunidades y dan atención a situaciones individuales en múltiples casos. Atendiendo activamente al $30 \%$ de la población (como se observa en la tabla 3 del tema 1.2 de este trabajo). Por ello es que una voz calificada para transmitir la esencia y promover las acciones de la agenda 2030 y los ODS es la del magisterio.

\subsection{Si las escuelas y los maestros son clave, el normalismo es su motor natural.}

Además de ser la más importante fuente de docentes preparados y calificados del país, las normales son una Alma Mater de apego y respeto para la mayor parte de sus estudiantes y egresados, son una casa a la que ven con respeto y cariño, a la que desean volver y son atentos a sus llamados. También, por sus esquemas de prácticas docentes, son una red de acercamiento y actualización para las escuelas, que suelen tomar con atención las enseñanzas que provienen de ellas y que suelen aprovechar para sus procesos didácticos. Dicha red se puede entender considerando el tipo de normales la cantidad que existen en el país (como se aprecia en la tabla 1 del tema 1.1 de este documento). Sin olvidar que muchas de las escuelas del país cuentan con al menos un egresado normalista. Es por esto que las escuelas normales son la voz autorizada y con capacidad de acción para cualquier meta donde se requiera a las escuelas y a sus docentes, por ello deben ser tomadas en cuenta, con un programa propio, para la búsqueda del cumplimiento de los ODS en nuestro país.

\section{Objetivos}

El objetivo general es aplicar un proyecto específico por parte de las escuelas normales de México, como parte del nivel de educación superior, para conocer, hacer propias y difundir los ODS, coadyuvando así a su correcto cumplimento. Alcanzar con ello un reposicionamiento social del sector y lograr una nueva cohesión del normalismo en el país. Puedo desglosar las siguientes metas.

- Conocer de forma real y especializada los ODS en la Educación Normal.

- Incorporar temáticas ODS a asignaturas de la educación normal.

- Desarrollar una vida normalista incluyente y participativa en los ODS.

- Difundir e implementar acciones de los ODS en la educación básica mediante el sistema de educación normal.

- Replicar temáticas y mecanismos ODS normalistas en escuelas de prácticas y egresados normalistas. 
- Revalorar la Educación Normal dentro de la Educación Superior.

- Reposicionar a los docentes como líderes sociales y resurgimiento de su función como agentes de cambios en sus comunidades.

- Firmar un pacto nacional de escuelas normales para un trabajo sistematizado, coordinado y colaborativo por el alcance de los ODS.

Cabe mencionar que, aprovechando el contexto de reforma del nivel en el país y de las leyes de educación, se busca que esta propuesta sea integrada como mecanismo de trabajo cotidiano. Para ello, poco a poco, se presenta este trabajo ante diferentes autoridades educativas planteando como muestra el pilotaje en nuestra escuela normal, que está próximo a iniciarse, que es la más influyente y reconocida en México.

\section{Como estamos con la instrumentación de la Agenda 2030 en México.}

En México, aparentemente, no se ha hecho mucho para el cumplimiento de la Agenda 2030 y de los ODS. Se puede ver en internet información sobre pocos eventos, artículos y estrategias escritas por parte, principalmente, del sector gobierno, cada dependencia desglosando su ODS [GOBIERNO DE MÉXICO. ESTRATEGIA NACIONAL PARA LA PUESTA EN MARCHA DE LA AGENDA 2030. México. https://www.gob.mx/cms/uploads/attachment/file/412433/Estrategia_Nacional_Implementacion_Age nda_2030.pdf].

En cuanto al nivel de enseñanza superior el máximo hecho es un congreso en el que se acuerda el implementar acciones en universidades para el logro de los ODS, sin que esto haya bajado realmente a las instituciones universitarias y se palpen estrategias al respecto. [COMPROMISO SOCIAL POR LA CALIDAD DE LA EDUCACIÓN (2016). México y los Objetivos del Desarrollo Sostenible 2030. <http://compromisoporlaeducacion.mx/mexico-y-losobjetivos-del-desarrollo-sostenible-2030/> ].

En el artículo oficial, por parte del gobierno federal, al respecto se menciona un evento logrado mediante la suma de todos los sectores del país por medio de una convocatoria nacional. Al respecto se puede apreciar que dicho esfuerzo no fue realizado como tal, y que, posiblemente, se cayó en un acto de simulación por cumplimiento o en una convocatoria específica para lograr emitir el documento [GOBIERNO DE MÉXICO. Agenda 2030, Objetivos de Desarrollo Sostenible. <https://www.gob.mx/agenda2030>]. Es visible que en estos procesos no fue considerado el sector normalista.

Tristemente solo de habla de acciones macro, no específicas, del sector gobierno, no se ve una inclusión directa de la sociedad civil, se le toma por naturaleza como beneficiaria, pero no se reconoce el papel y motor que puede llegar a ser para una puesta en marcha real [INSTITUTO NACIONAL DE ESTADISTICA Y GEOGRAFIA (INEGI) (2018). Sistema de Información de los Objetivos de Desarrollo Sostenible. < http://agenda2030.mx/\#/home>].

En el planteamiento oficial de la Agenda 2030 para México se mencionan organismos de seguimiento e implementación en cada una de las 32 entidades federativas del país, pero decepcionantemente no se sabe de ellos, ni funciones, ni conformación, ni ubicación.

También dicho escrito se presume como resultado un acopio de ideas, expresiones y textos de diversos sectores, pero lo cuantifican y se muestra como un pobre trabajo o convocatoria, ya que se menciona como producto de 438 comentarios y 14 documentos. 
En referencia al ODS 4 se plantean diferentes medidas muy generales para su cumplimiento, pero desafortunadamente México requiere una mayor inversión real, directa y transparente en su sistema educativo, y esto no se menciona, no se hace una propuesta específica de inversión directa, lo que deja entrever que será la continuidad del gasto bajo y mediante los procedimientos acostumbrados

Por lo anterior, en un análisis totalmente personal, asumo que en México no se ha realizado un proceso a fondo y específico para el correcto trabajo y logro de los ODS, aparentemente se optó por la comodidad de eventos y escritos que simulan y son generados para dar la impresión de cumplimiento, o de estar en el proceso de ello.

\section{La propuesta.- Hacerlo en casa para poder ser ejemplo y ayudarle al vecino}

En todo proyecto muchas veces grandes metas son alcanzadas por medio de la constancia y trabajo de actividades sencillas, generando una suma constante potencializadora. Esta propuesta va en este sentido, acciones puntuales, específicas y sencillas a realizar por las escuelas normales, hacia su interior y al exterior de los planteles.

Para el desarrollo y puesta en marcha de esta propuesta es necesario realizar un pilotaje nacional, empleando a una normal por estado, abarcando a las 32 entidades, para que estas posteriormente se conviertan en tutoras de las demás en una puesta en marcha ya general. Con esto convertir a este sector de la educación superior como receptor y difusor de los trabajos por los ODS [TAHL KESTIN, COORD. DE PROYECTO (SDSN AUSTRALIA/PACIFIC \& MONASH SUSTAINABLE DEVELOPMENT INSTITUTE, MONASH UNIVERSITY). AUTORES VARIOS. (2017). CÓMO EMPEZAR CON LOS ODS EN LAS UNIVERSIDADES. Australia. http://reds-sdsn.es/wp-content/uploads/2017/02/Guia-ODSUniversidades-1800301-WEB.pdf]

En este sentido, como lo mencioné anteriormente, en la Benemérita Escuela Normal Veracruzana, principal escuela de educación normal de México, ya se prepara la adecuación de actividades a desarrollar para la aplicación del proyecto, además de que de la mano se alistan los mecanismos para que esta dinámica sea considerada como metodología a aplicar en todo el subsistema por medio de la reingeniería normativa del sector en México.

Metas planteadas en los ODS no se logran inmediatamente, por lo que se requieren unos pocos años de esfuerzo para poder tener resultados tangibles, al menos tres años para tener parámetros medibles de alcances en el contexto nacional.

Síntesis de acciones a seguir en la propuesta:

- Acercamiento de la temática ODS a las escuelas Normales involucradas, esto con personal verdaderamente calificado y especializado en el tema. Esta impartición de contenidos deberá ser a toda persona del nivel, desde autoridades hasta personal de servicios, pasando por alumnos. Será de forma detenida y profesional, no solo un espacio o una conferencia para cumplir.

- Cada escuela normal realizará el planteamiento de actividades que se llevarán a cabo para incluir los ODS en todo proceso escolar, desde funciones 
administrativas hasta servicios. Es importante que no solo se considere el ODS 4, que por naturaleza se le dará principal importancia, sino que la escuela especifique al menos una acción tangible y comprobable para cada uno de los ODS.

\begin{tabular}{|c|c|}
\hline ODS & Posible Actividad a Realizar \\
\hline Fin de la pobreza & $\begin{array}{l}\text { Realizar un censo al interior del plantel para analizar situaciones } \\
\text { socioeconómicas y con esto buscar asignación de becas de trabajo, } \\
\text { excelencia y desempeño. }\end{array}$ \\
\hline Hambre cero & $\begin{array}{l}\text { Basados en el censo de ODS anterior determinar que miembros de la } \\
\text { comunidad son susceptibles a apoyo de becas alimenticias por parte } \\
\text { de la cafetería o venta escolar. }\end{array}$ \\
\hline Salud y bienestar & $\begin{array}{l}\text { Generar una práctica deportiva obligada comprobable al interior de } \\
\text { las instalaciones escolares, esto mediante clubes deportivos, de una } \\
\text { hora diaria, y que esto sea parte del currículo de toda persona de la } \\
\text { escuela. Esto en el mejor de los casos con acompañamiento de } \\
\text { índices de salud y evolución. }\end{array}$ \\
\hline $\begin{array}{l}\text { Educación } \\
\text { calidad }\end{array}$ & $\begin{array}{l}\text { - Fortalecimientos de procesos de enseñanza aprendizaje. } \\
\text { - Sistema estadístico de resultados escolares. } \\
\text { - Incorporación real de docente tutor o acompañante por cada } \\
\text { aula o materia del plantel. }\end{array}$ \\
\hline Igualdad de género & $\begin{array}{l}\text { Análisis de todas las prácticas y procesos realizados al interior del } \\
\text { platel, esto por parte de toda la comunidad escolar, con el fin de } \\
\text { detectar alguna posible diferencia existente derechos y obligaciones } \\
\text { derivadas por género, a la par de colectivamente presentar propuestas } \\
\text { para su corrección. }\end{array}$ \\
\hline $\begin{array}{l}\text { Agua limpia } \\
\text { saneamiento }\end{array}$ & $\begin{array}{l}\text { Diseñar sistemas de captación y purificación de agua pluvial en el } \\
\text { plantel, para su posterior reutilización. }\end{array}$ \\
\hline Energía asequible & $\begin{array}{l}\text { Realizar un análisis de todo los espacios de la escuela, cada persona o } \\
\text { grupo su área, con el fin de detectar fugas o alteraciones de energía, } \\
\text { ubicar y eliminar (sustitución) de aparatos de alto consumo } \\
\text { energético, y el cambio de luminarias por sistemas ahorradores. }\end{array}$ \\
\hline $\begin{array}{l}\text { Trabajo decente y } \\
\text { crecimiento } \\
\text { económico }\end{array}$ & $\begin{array}{l}\text { Toda escuela tiene personas que realizan venta de artículos o } \\
\text { prestación de servicios. Resultaría interesante realizar un censo al } \\
\text { respecto para convertirlo en un listado de conocimiento del plantel } \\
\text { incentivando la compra o utilización de lo ofertado por miembros de } \\
\text { la comunidad, evitando la derrama se vaya a otro lado y fortaleciendo } \\
\text { la interior. }\end{array}$ \\
\hline $\begin{array}{l}\text { Industria, } \\
\text { innovación } \\
\text { infraestructura }\end{array}$ & $\begin{array}{l}\text { Las escuelas normalmente tienen necesidades a atender en su } \\
\text { infraestructura, pero también cuentan con espacios atractivos para } \\
\text { realizar actividades de otras índoles para la sociedad. La comunidad } \\
\text { puede detectar estos espacios, organizar esquemas de trabajo y rentar } \\
\text { esporádicamente espacios con el fin de obtener ingresos que ayuden } \\
\text { a la manutención escolar. }\end{array}$ \\
\hline Reducción de las & Acercarse a otras instituciones afines o hermanas con la intención de \\
\hline
\end{tabular}




\begin{tabular}{lll}
\hline desigualdades & $\begin{array}{l}\text { establecer lazos de cooperación, compartiendo experiencias y } \\
\text { generando sinergias que permitan el progreso de ambas mediante la } \\
\text { bilateralidad. }\end{array}$ \\
$\begin{array}{l}\text { Ciudades } \\
\text { comunidades } \\
\text { sostenibles }\end{array}$ & $\begin{array}{l}\text { Proyectar y desarrollar diferentes actividades en las escuelas con el } \\
\text { fin de transformarlas en sedes incluyentes seguras donde sus } \\
\text { integrantes puedan resolver sus necesidades. }\end{array}$ \\
$\begin{array}{l}\text { Producción } \\
\text { consumos } \\
\text { responsables }\end{array}$ & $\mathrm{y}$ & $\begin{array}{l}\text { Promover hortalizas grupales, realizando eventos de prueba y } \\
\text { demostración de cosechas. }\end{array}$
\end{tabular}

$\begin{array}{ll}\text { Acción por el clima } & \begin{array}{l}\text { Buscar espacios deforestados, obtener el apoyo del gobierno con } \\ \text { árboles endémicos del lugar para sembrar y realizar diversas } \\ \text { actividades de siembra de árboles. }\end{array} \\ \text { Vida submarina } & \begin{array}{l}\text { Detectar especies afectadas lo más cercanas a su centro escolar o } \\ \text { entorno, identificando causas y motivos para generar campañas de } \\ \text { concientización. }\end{array}\end{array}$

Vida de ecosistemas Identificar la biodiversidad de su región, promover su conocimiento terrestres $\quad$ social y establecer acciones para la sociedad con las que se le pueda dar el cuidado necesario para su preservación.

Paz, justicia e En especial México atraviesa una crisis severa de inseguridad. Se instituciones sólidas puede realizar, aprovechando redes sociales, algún medio para la comunidad escolar que de alertas, tips de seguridad y permita la ubicación o localización de sus integrantes.

$\begin{array}{ll}\text { Alianza para lograr } & \text { Identificar los niveles de gobierno, empresas o sociedad en general } \\ \text { objetivos } & \text { que pueda realizar algún aporte, de cualquier tipo, para el } \\ & \text { cumplimiento de los ODS en las escuelas, y acordar un apoyo } \\ & \text { recíproco o un sistema de intercambio de acciones en pro de los ODS } \\ & \text { de cada institución. }\end{array}$

Tabla: Ejemplos de acciones a proponer y emprender en escuelas normales en pro de los ODS a partir de su comunidad.

- La puesta en marcha de esta propuesta será por partes, dejando para el semestre inicial el proceso de actividades vivenciales para la escuela, para que la comunidad refuerce su conocimiento y vaya adoptando prácticas cotidianas encaminadas al cumplimiento de los ODS.

- Una vez generado el sistema vivencial al interior de las escuelas normales, al siguiente semestre se trasladarán tanto conocimientos y actividades a las escuelas de práctica docente en las que tengan alcance las normales.

- En un tercer semestre, se procederá a reuniones, cursos, capacitaciones y talleres con egresados normalistas para transmitir y promover de igual manera conocimientos y actividades para que lo apliquen y desarrollen en sus escuelas. Además de capacitación se deberá brindar acompañamiento. 
- Durante cada semestre se harán mediciones iniciales, intermedias y finales para poder tener referencias reales del alcance del proyecto y así, a la repetición de procesos, tener en claro actividades a continuar, cambiar o modificar.

- Una vez aplicado el pilotaje de los tres semestres, se procederá a la puesta en marcha en todas las escuelas normales del país. Tomando como base las actividades de ejemplo (tabla 4 tema 4 ).

- Previo al desarrollo de la metodología en el país, se firmará un pacto nacional de escuelas normales para un trabajo sistematizado, coordinado y colaborativo por el alcance de los ODS. Integrando a toda la comunidad normalista (tabla 2 tema 1.1)

- Afortunadamente esta metodología puede resultar económica en aplicación, es viable que planteles lo operen con recursos propios, sin generar gastos adicionales.

- Es importante involucrar al gobierno federal y a gobiernos estatales para poder obtener incentivos para las escuelas que participen.

- Se deberá sumar todos los resultados de la metodología al esfuerzo nacional por los ODS y al informe final que este realice.

En este punto es importante reafirmar que la innovación como tal, por sencilla que parezca, es la real aplicación y difusión de actividades para el cumplimiento de los ODS, y así la réplica social de estas prácticas. No se buscan actividades complejas o instructivos estrictos, se pretende el entendimiento y aceptación de la Agenda 2030 y los ODS, y con ello el realizamiento, a corde a capacidades, realidades y recursos de cada plantel, de acciones cotidianas que involucren todos los ODS, con esto convertirlo en una práctica sostenible de comunidades, adecuando prácticas para un mejoramiento del entorno social.

En mi opinión innovar no siempre es descubrir soluciones nuevas a retos o problemas, es crear caminos certeros para fines específicos o globales, con mecanismos sencillos o complejos, pero con meta fija y cumplible.

\section{Posibles obstáculos.}

Como en toda actividad laboral es altamente probable que se presenten inconvenientes que dificulten la aplicación de la propuesta, entre los que destacan los siguientes:

- Incompatibilidad de propuesta con políticas gubernamentales.

- Desinterés de autoridades por impulsar propuesta.

- Burocracia.

- Propuesta no interesante por no reflejar beneficios políticos.

- Desanimo por parte de escuelas normales por desarrollar metodología.

- Baja respuesta al interior de normales, con miembros de la comunidad.

- Apatía por parte de escuelas de práctica y egresados.

\section{Resultados esperados.}

- Correcta y real difusión de esfuerzos globales por la Agenda 2030 y ODS.

- Inclusión del normalismo es estos esfuerzos por parte del país. 
- Suma de la sociedad en general en estos trabajos.

- Valoración de las escuelas normales en su papel de difusión de políticas públicas.

- Ser el país con más logros en ODS por parte del nivel de enseñanza superior.

- Convergencia de contenidos académicos y temática ODS.

- Reposicionamiento de docentes en su liderazgo social.

- Utilización de recursos y redes existentes en propósitos nacionales.

- Lograr que México sea una de las naciones con más avances en ODS.

- Verdadero cumplimiento de pactos internacionales del país.

- Realizar procesos incluyentes sociedad-gobierno.

- Tomar a las escuelas como principal canal para valoración, aplicación y desarrollo de políticas públicas.

\subsection{Formato de medición de resultados.}

El siguiente formato pretende facilitar la explicación y vaciado de datos a los encargados de actividades, siendo tambien un mecanismo rápido de recolección de información en los lugares donde se aplique la metodología, siendo este muy sencillo de realizar.

\begin{tabular}{|l|l|}
\hline ESCUELA & \\
\hline RESPONSABLE DE APLICACIÓN & \\
\hline ÁREAS INVOLUCRADAS & \\
\hline POBLACIÓN DIRECTA PARTICIPANTE & \\
\hline POBLACIÓN INDIRECTA PARTICIPANTE & \\
\hline
\end{tabular}

\begin{tabular}{|c|c|c|c|c|c|c|c|c|c|c|c|c|c|c|c|c|}
\hline \multirow{2}{*}{ No. } & \multirow[t]{2}{*}{ ACTIVIDAD PROPUESTA } & \multirow{2}{*}{ ODS } & \multirow{2}{*}{ PERIODO } & \multirow{2}{*}{ INVOLUCRADOS } & \multicolumn{3}{|c|}{ ACEPTACIÓN } & \multicolumn{4}{|c|}{ CUMPLIMIENTO } & \multicolumn{3}{|c|}{ GUSTO } & \multicolumn{2}{|c|}{ PRÁCTICA COT. } \\
\hline & & & & & B & M & $\mathbf{A}$ & $25 \%$ & $50 \%$ & $75 \%$ & $100 \%$ & B & M & $\mathbf{A}$ & SI & No \\
\hline 1 & & & & & & & & & & & & & & & & \\
\hline 2 & & & & & & & & & & & & & & & & \\
\hline
\end{tabular}

\begin{tabular}{|c|c|c|c|}
\hline ACT. & DESCRIPCIÓN CONTEXIO APLICACIÓN & ACT. & ACTITUD ANTES Y DESPUES PARTICIPANTES \\
\hline & & & \\
\hline & & & \\
\hline ACT. & LOGROS ESPECIFICOS ACTIVIDADES EN PLANTEL & ACT. & DIFICULTADES ENCONTRADAS \\
\hline & & & \\
\hline & LOGROS ESPECIFICOS EN COMINIDAD & ACT. & OBSERVACIONES Y COMENTARIOS GENERALES \\
\hline ACT. & & & \\
\hline & & & \\
\hline & & & \\
\hline
\end{tabular}

\section{Conclusiones.}

La más importante conclusión es la confirmación de la certeza de que la educación es el camino para todo cambio y mejoramiento social en las naciones, que tristemente es echa a 
un lado por no contar con autoridades que conozcan su verdadero valor, o simplemente por preferir una sociedad no tan preparada para evitar así una exigencia mayor a la autoridad por parte de su pueblo. Sin duda alguna puedo afirmar que también es tarea de las escuelas, en este caso de las normales, recuperar terreno y valor. El normalismo no es algo obsoleto, por su carácter social es totalmente vigente, solo se debe buscar la convergencia y mecanismos para el trabajo conjunto del nivel, el gobierno y la sociedad. El sector educativo puede ayudar a hacer frente a problemáticas o compromisos de cualquier índole, puede ser el mayor capital de una nación.

Toda política pública o programa que no sea correcta y debidamente bajada a la sociedad está prácticamente condenada al fracaso.

En cuanto corresponde al país, aparentemente el trabajo por la agenda 2030 solo ha sido de escritorio, estamos a tiempo de cambiar, mejorar y lograr metas, solo es cuestión de trabajar de manera real y verdaderamente integrar a todos los sectores de la sociedad, cada uno a partir de sus acciones naturales en su círculo de influencia.

La enseñanza superior es la cima de un proceso educativo, el que puede generar individuos con experiencias y conocimientos necesarios para lograr cambios sociales, no debe llevarse este sector a la simulación, hacerlo lo condenaría a limitar sus habilidades naturales y a la eliminación del pensamiento crítico creativo que puede dar soluciones aplicables al país.

La agenda 2030 y los ODS son importantes, trascendentes en la vida de todo habitante del planeta. Aún es tiempo, se está en un lapso en el que todavía se puedan proponer y poner en marchas diferentes acciones para buscar su cumplimiento.

Los perfiles y esquemas educativos dan a una nación perspectiva, pero la que lo valora, desarrolla y explota tiene el capital más redituable e inagotable del planeta.

\section{Referencias.}

COMPROMISO SOCIAL POR LA CALIDAD DE LA EDUCACIÓN (2016). México y los Objetivos del Desarrollo Sostenible 2030. <http://compromisoporlaeducacion.mx/mexico-y-losobjetivos-del-desarrollo-sostenible-2030/> [Consulta: 22 de marzo de 2019].

GOBIERNO DE MÉXICO. ESTRATEGIA NACIONAL PARA LA PUESTA EN MARCHA DE LA AGENDA 2030. México.

$<$ https://www.gob.mx/cms/uploads/attachment/file/412433/Estrategia_Nacional_Implementacion_Ag enda_2030.pdf> [Consulta: 22 de marzo de 2019].

GOBIERNO DE MÉXICO. Agenda 2030, Objetivos de Desarrollo Sostenible. $<$ https://www.gob.mx/agenda2030> [Consulta: 22 de marzo de 2019].

INSTITUTO NACIONAL DE ESTADISTICA Y GEOGRAFIA (INEGI) (2018). Sistema de Información de los Objetivos de Desarrollo Sostenible. $<$ http://agenda2030.mx/\#/home> [Consulta: 22 de marzo de 2019].

ONU MUJERES (2015). La Agenda 2030 para el Desarrollo Sostenible. $<$ http://www.unwomen.org/es/what-we-do/post-2015> [Consulta: 22 de marzo de 2019]. 
SECRETARÍA DE EDUCACIÓN PÚBLICA (SEP). Sistema Interactivo de Consulta Estadística Educativa. <https://www.planeacion.sep.gob.mx/principalescifras/> [Consulta: 22 de marzo de 2019].

TAHL KESTIN, COORD. DE PROYECTO (SDSN AUSTRALIA/PACIFIC \& MONASH SUSTAINABLE DEVELOPMENT INSTITUTE, MONASH UNIVERSITY). AUTORES VARIOS. (2017). CÓMO EMPEZAR CON LOS ODS EN LAS UNIVERSIDADES. Australia.

$<$ http://reds-sdsn.es/wp-content/uploads/2017/02/Guia-ODS-Universidades-1800301-WEB.pdf $>$

[Consulta: 22 de marzo de 2019].

VERONICA MEDRANO CAMACHO, EDUARDO ÁNGELES MENDEZ, MIGUEL ÁNGEL MORALES HERNANDEZ. (2017). LA EDUCACIÓN NORMAL EN MÉXICO, ELEMENTOS PARA SU ANÁLISIS. México.

$<$ http://publicaciones.inee.edu.mx/buscadorPub/P3/B/108/P3B108.pdf $>$ [Consulta: 22 de marzo de 2019]. 